ARTICLE

\title{
Modeling of H(n,n) Recoil Proton Injection into LWR Fuel Cladding with Sequential Use of MCNP and SRIM Codes
}

\author{
Yasushi NAUCHI ${ }^{*}$ and Takanori KAMEYAMA \\ Central Research Institute of Electric Power Industry, 2-11-1 Iwado Kita, Komae-shi, Tokyo, 201-8511, Japan
}

\begin{abstract}
Neutron elastic scattering reactions with hydrogen in moderator of a light water reactor are simulated and scored event by event with the MCNP-5 code. With the data, the initial energy and flying direction of the recoil protons in the reactions are deduced and input to the SRIM-2008 code. Finally, injection of the protons into cladding by the recoiling process is quantified. The amount of hydrogen accumulated in the cladding by the process at burn-up of $40 \mathrm{MWd} / \mathrm{kgHM}$ reaches 5 12 wt-ppm, which corresponds to $5 \sim 20 \%$ of hydrogen content measured for conventional irradiated fuel claddings that are intact.
\end{abstract}

KEYWORDS: recoil proton, hydrogen absorption, LWR fuel cladding, MCNP-5, SRIM-2008

\section{Introduction}

Extension of fuel burn-up is progressed in the world to reduce the number of spent fuel assemblies. Currently, the maximum discharge burn-up of assembly is limited to $55 \mathrm{MWd} / \mathrm{kgHM}$ in Japan. For further extension, fuel integrity must be assured up to the target burn-up.

Increment of hydrogen $(\mathrm{H})$ content in claddings is one of the most important issues constraining the burn-up extension since it makes the claddings brittle. Up to burn-up of $40 \mathrm{MWd} / \mathrm{kgHM}$, absorption rate of $\mathrm{H}$ in zirconium ( $\mathrm{Zr}$ ) alloy is low and its content is less than 50 100 wt-ppm in a boiling water reactor (BWR). ${ }^{1)}$ The rate increases for burn-up exceeding $50 \mathrm{MWd} / \mathrm{kgHM}$. Extensive studies have been conducted to clarify the mechanisms of the absorption behavior to prevent the $\mathrm{H}$ embrittlement and fuel failure caused by the $\mathrm{H}$ absorption at even higher burn-up.

The $\mathrm{H}$ absorption mechanisms have been mainly investigated from viewpoints of chemical corrosion. By out of pile tests, correlation has been found between thickness of oxide layer on outer surface of the fuel rods and the hydrogen content in the Zircaloy claddings. Accordingly, they consider major process of the $\mathrm{H}$ absorption is

$$
2 \mathrm{H}_{2} \mathrm{O}+\mathrm{Zr} \rightarrow \mathrm{ZrO}_{2}+4 \mathrm{H}
$$

in reactor coolant water (moderator) and sequential absorption of the extra $\mathrm{H}$ atoms in the Zircaloy. Radical species yielded by ionization of $\mathrm{H}_{2} \mathrm{O}$ by $\gamma$ rays and neutrons are also considered to enhance the $\mathrm{H}$ absorption process described above. However, injection of recoil protons by elastic scattering of neutron with hydrogen in water has been seldom considered although a considerable number of them are radiated in the moderator as described in Section IV. 2. In this paper, we focus on the proton transport and quantify its effect on the $\mathrm{H}$ absorption in claddings with simplified models.

*Corresponding author, E-mail:nauchi@criepi.denken.or.jp

\footnotetext{
Corresponding author, E-mail.nauchi@criepi.denken.or.jp
}

(c) 2011 Atomic Energy Society of Japan, All Rights Reserved.

\section{Hydrogen Absorption in Cladding by Elastic Scattering of Neutron with Hydrogen}

In LWRs, fast neutrons are radiated by fission reactions. Energy of them is $2 \mathrm{MeV}$ on average. They lose their energy through scattering processes with nuclides in the core. The major process of the neutron moderation is the elastic scattering with hydrogen in the coolant water, since an incident neutron loses its $50 \%$ of kinetic energy by the scattering on average. The transferred energy to the $\mathrm{H}$ atom exceeds the bond energy of water molecule $(\sim 5 \mathrm{eV})$ so that it becomes a proton ion. The proton moves in the moderator and loses its energy by the ionization and the excitation processes and by the Rutherford scatterings. Protons of $1 \mathrm{MeV}$ can move by $0.1 \sim 0.2 \mathrm{~mm}$ (depending on void ratio) in the moderator. Accordingly, some of them can penetrate into a cladding through the moderator (see Fig. 1).

It is noteworthy that one neutron of $2 \mathrm{MeV}$ can generate 10 recoil protons on average as described in Section IV. 2. Since roughly 2.5 neutrons are radiated per fission, the number of recoil proton emissions is expected to be 25 times

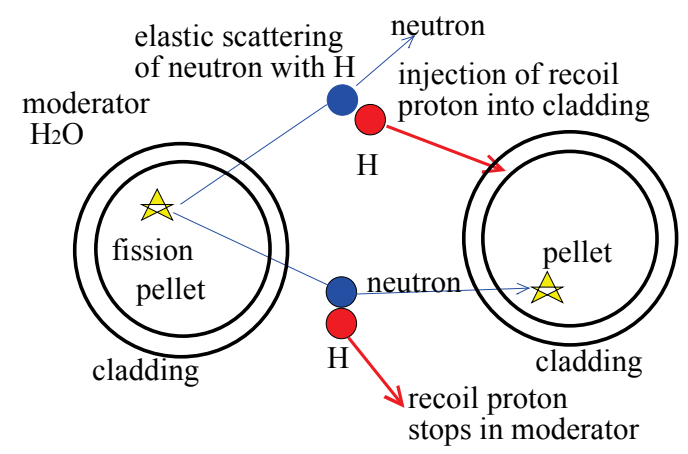

Fig. 1 Schematic view of recoil proton injection into cladding through elastic scattering process of neutron with hydrogen in moderator 
larger than that of nuclides lost by fission in fuel pellets. Accordingly, we decided to quantify the number of proton injections into the claddings by the process.

\section{Quantification Procedures}

In this chapter, we establish a simulation method of the injection of the recoil protons into the claddings. The transport of neutrons initiated by the fission, the neutron collision with $\mathrm{H}$, and the transport of the recoil protons are simulated in a simplified geometry.

\section{Components Considered in Model Calculation}

Japanese current BWR cladding is Zircaloy-2 lined by metal zirconium on its inner surface. In normal operations, $\mathrm{Zr}$ on the outer surface of an irradiated fuel cladding is oxidized and $\mathrm{ZrO}_{2}$ layer of tens $\mu \mathrm{m}$ thickness is formed. The cladding is further covered by hard and soft cruds made out of water, oxygen, metals, etc. The oxide layer and the cruds have some roles in $\mathrm{H}$ absorption in claddings by chemical processes, but they do not have significant effects on the $\mathrm{H}(\mathrm{n}, \mathrm{n})$ reactions in the moderator because there are no strong neutron absorbers in them. Besides, the thickness of the layer and the cruds are thin so that the number of nuclear reactions occurring in them is even smaller than that in the fuel, the cladding, and the moderator. At the first step of this study, we considered only fresh fuel cases in which the layer and the cruds do not exist.

In this paper, a simplified unit cell of BWR geometry (high burn-up, $9 \times 9$ type) was used. Schematic view of geometry is illustrated in Fig. 2. Detailed specifications are in Table 1. The ${ }^{235} \mathrm{U}$ enrichment is the averaged one of BWR fuel assemblies, while pellets with several kinds of ${ }^{235} \mathrm{U}$ enrichment are loaded in the commercial assemblies. ${ }^{2)}$ The cladding was modeled by $\mathrm{Zr}$ metal. Outer boundaries of vertical and lateral directions were set reflection for neutron transport calculation. Accordingly, vertical (z-axis) distribution of $\mathrm{H}(n, n)$ reaction is calculated flat in the geometry. The model is equivalent to infinite one in the vertical direction. Void effects of moderator were considered as described in Section III. 3. Burn-up effects of isotopic composition in the fuel pellet were also neglected although they are discussed in Section IV. 3.

\section{Sequential Calculations with MCNP-5 and SRIM-2008}

We performed transport calculations of neutrons and pro-

Table 1 Specification of components used for the calculation

\begin{tabular}{lccc}
\hline components & fuel & cladding & moderator \\
\hline material & $\begin{array}{c}\mathrm{UO}_{2} \\
4 \%{ }^{-2}{ }^{235} \mathrm{U} / \mathrm{U}\end{array}$ & $\mathrm{Zr}$ & $\begin{array}{c}\mathrm{H}_{2} \mathrm{O} \\
0,40,70 \% \text { void ratio }\end{array}$ \\
\hline $\begin{array}{l}\text { geometry } \\
\text { outer diameter* } \\
\text { cell pitch }\end{array}$ & $0.98 \mathrm{~cm}$ & $1.12 \mathrm{~cm}$ & \\
\hline temperature & $900 \mathrm{~K}$ & $559 \mathrm{~K}$ & $559 \mathrm{~K}$ \\
\hline & $*$ neglecting gaps between fuel and cladding
\end{tabular}

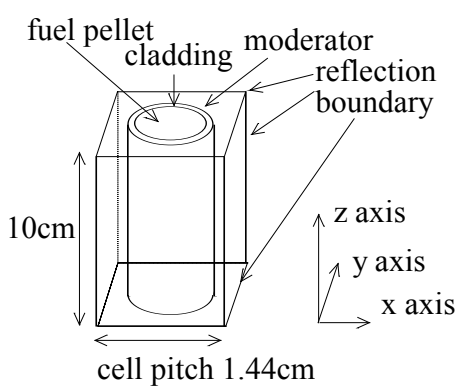

Fig. 2 Simplified BWR fuel cell geometry for the calculation

tons separately to estimate energy, direction, and tracks of a recoil proton penetrating into the cladding one by one. The neutron transport was calculated with the continuous energy Monte Carlo code MCNP- $5^{3)}$ and the proton transport was done with the SRIM-2008 code. ${ }^{4)}$

(1) MCNP-5 Calculation

We made an MCNP cross section library with the NJOY99 code $^{5)}$ based on JENDL-3.3 ${ }^{6)}$ taking the temperature of the materials in the Table 1 into account. With MCNP-5 and the library, we performed eigenvalue calculations in the analog mode. 50 generations of calculations were done to obtain the converged neutron source distribution. $1,000,000$ neutrons were employed per generation, nominally. The source was written in a file with the surface source write function.

Then we continued the neutron transport calculation in the 51 st generation with the surface source read function. In the calculation, we wrote down the every collision event in the moderator to a file with the ptrac function of MCNP-5. In the calculation, transport of a neutron was terminated within the generation with the nonu option. By the procedure, reaction type, reaction location $\boldsymbol{r}$, direction $\boldsymbol{u}$, and energy $E$ of incident $\left(_{\text {in }}\right)$ and outgoing $\left(_{\text {out }}\right)$ neutrons were stored for every collision. At the same time, the number of fissions induced by the neutrons in the generation was tallied.

If we do the calculation described above at once, neutron collision events in generations less than or equal to 50 are also written down to the file with ptrac. We can not distinguish events in the 51st generation among the data written in the file. Accordingly, the two steps calculations mentioned above were done.

(2) Proton Energy Calculation

We selected the neutron collisions with $\mathrm{H}$ written in the file by ptrac. Some lower energy neutrons inelastically collide with hydrogen atoms in water molecules. Among those low energy neutrons, some ones radiate protons, but others are given thermal energy by the molecules. Thus the ionization process in the lower energy region is so complicated. We neglected the inelastic scattering in this paper.

For an elastic scattering event, we estimated the initial energy $E_{p}$ and the direction $\boldsymbol{u}_{\mathrm{p}}$ of the recoil proton by the conservation lows of the energy and the momentum neglecting the chemical bond energy:

$$
E_{\text {in }}=E_{\text {out }}+E_{p},
$$




$$
\sqrt{\frac{2 E_{\text {in }}}{m_{n}} \mathbf{u}_{\text {in }}}=\sqrt{\frac{2 E_{\text {out }}}{m_{n}} \mathbf{u}_{\text {out }}}+\sqrt{\frac{2 E_{p}}{m_{p}}} \mathbf{u}_{p}
$$

Here subscripts $n$ and $p$ mean the neutron and the proton, respectively. $m$ means the mass of the proton or the neutron.

(3) SRIM-2008 Calculation

Transport of the protons in the moderator is calculated stochastically with SRIM-2008 in which interaction of an ion and materials in media is approximated by binary-collision model. ${ }^{4)}$ In SRIM-2008, the energy loss processes of charged particles by i) the excitation and the ionization and ii) the Rutherford scattering are considered. Accordingly, linear tracks of ions by process i) and non-linear random ones by process ii) can be both simulated.

Prior to the SRIM calculations, the nearest distance from the cladding surface to the $H(n, n)$ reaction point was calculated for each proton. In the SRIM-2008 package, ion range in a material is provided as a function of its energy. If the distance is longer than the range, the proton will stop in the moderator. We discriminated those protons to reduce calculation time with the SRIM-2008 code. In the discrimination, straggling of the range was taken into account. By the procedure, $0.2 \sim 0.7 \%$ of the $\mathrm{H}(\mathrm{n}, \mathrm{n})$ reactions were selected.

In the 3-D transport calculation of the protons with SRIM-2008, we can input the initial position, energy, and direction of a proton one by one, although multi-layered slabs geometry of media perpendicular to $\mathrm{X}$-axis (as shown in Fig. 3, bottom figure) is only allowed. We made calculation models of SRIM-2008 from 3D - MCNP-5 geometry. In the MCNP-5 geometry (Fig. 2), we drew a ray from the reaction point $\boldsymbol{r}$ along with the initial direction $\boldsymbol{u}_{p}$ of each proton calculated in Section III. 2. (2). In the drawing, the reflection boundaries were removed so that some rays may extend further. Then we evaluated the nearest (or cross) point $\boldsymbol{r}_{\text {near }}$ on the ray to the cladding. In Fig. 3, $\boldsymbol{r}_{\text {near }}$ of the particle " 1 " is $H(n, n)$ reaction point $\boldsymbol{r}_{1}$ since the particle goes far from the cladding along with $\boldsymbol{u}_{p 1}$. Contrarily, the ray of particle " 2 " from $\boldsymbol{r}_{2}$ intersects surface of the cladding so the $\boldsymbol{r}_{\text {near, } 2}$ is at the intersection point.

We rotated the geometry on the $\mathrm{x}-\mathrm{y}$ plane around the origin $O$ and displaced it one by one so that the transformed $\boldsymbol{r}_{\text {near }}, \boldsymbol{r}_{\text {near }}{ }^{\prime}=\left(x_{\text {near }}, y_{\text {near }}, z_{\text {near }}{ }^{\prime}\right)$, locates on the $x$-axis $\left(y_{\text {near }}{ }^{\prime}=0, z_{\text {near }}{ }^{\prime}=0\right)$ as shown in Fig. 3. $\boldsymbol{u}_{p}$ was also rotated. Then, we approximated the curbed geometry of the cladding and the moderator near the $\mathrm{x}$-axis by 2 - layered slabs in the SRM-2008 geometry. The information of the linear transformation (the rotation and the displacement) was stored for each recoil proton.

Then the transport of each proton with the initial energy $E_{p}$ flying in direction of $\boldsymbol{u}_{\boldsymbol{p}}$ ' was simulated with SRIM-2008 and the position of proton stop, $\boldsymbol{r}_{\text {stop }}$, was deduced. Then the inverse transformation was performed for $\boldsymbol{r}_{\text {stop }}$ ' to obtain the actual stopping position of the proton, $\boldsymbol{r}_{\text {stop }}$. We counted the number of protons stop in the cladding. The vertical (z-axis) position of $\boldsymbol{r}_{\text {stop }}$ may be out of $10 \mathrm{~cm}$ vertical height of MCNP calculation cell in Fig. 2. In this study, we assumed vertically outgoing current of proton is equivalent to incom-
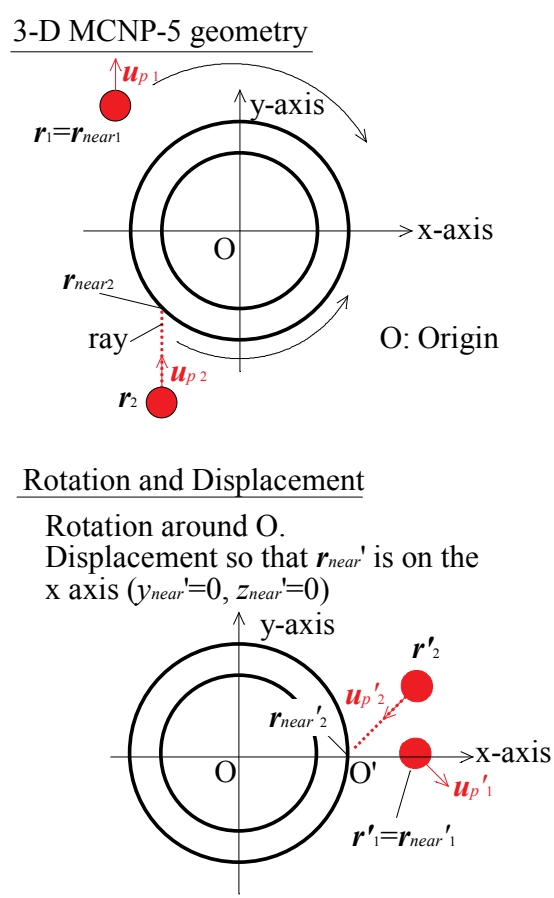

multi layered slabs SRIM-2008 geometry

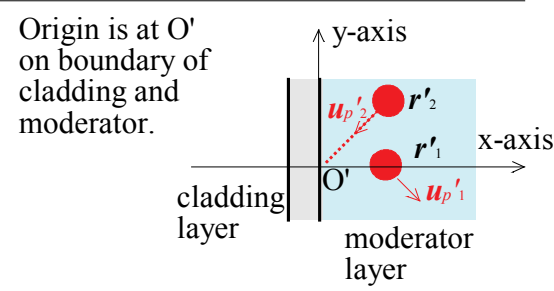

Fig. 3 pproximation of 3-D MCNP-5 geometry by multi-layered slabs SRIM-2008 one

ing one (vertically infinite model).

By comparing the number with the tallied fission rate in the generation, increment of $\mathrm{H}$ content in cladding per cell power through these focused processes was obtained.

\section{Modeling of Moderator}

In BWR, the void ratio of the coolant increases from $0 \%$ to $70-80 \%$ as the coolant elevates. The ratio may strongly affect the transports of the neutrons and the protons. Then we surveyed the increment of $\mathrm{H}$ content in the cladding in relation to the ratio.

In two-phase flow, distribution of the vapor and the liquid phases changes from the bubbly flow to the slug and the annular flow as the void ratio increases. Rigorous modeling of the two-phase coolant is so complicate and not practical. In this paper, we modeled the moderator in two ways: 1) homogeneous flow and 2) annular flow models (see Fig. 4). In the latter, a cylindrical liquid film coaxially covers a fuel rod.

In the homogeneous model, density of moderator $\rho_{m}$ is calculated by averaged ones of the vapor, $\rho_{v}$ and the liquid, $\rho_{l}$, weighted by volume ratio $(V R)$ of them.

$$
\rho_{m}=V R \times \rho_{v}+(1-V R) \times \rho_{l} .
$$

Stopping powers of the liquid and the vapor phases are dif- 


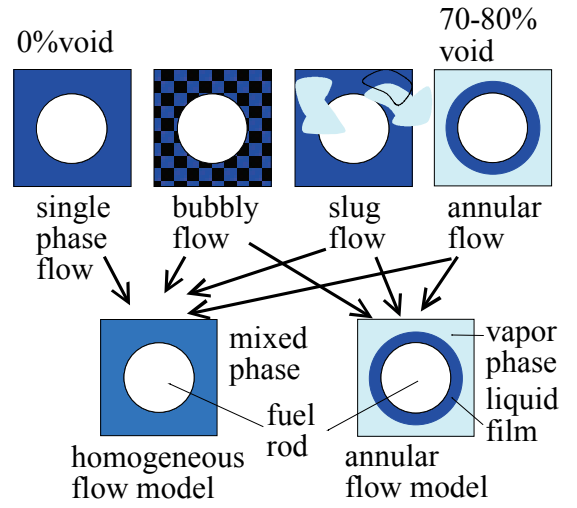

Fig. 4 Modeling of two-phase moderator flow

ferent. We performed two separate calculations with SRIM-2008 using the either stopping powers $\left((\mathrm{dE} / \mathrm{dx})_{\mathrm{v} \text { or } 1)}\right.$ multiplied by density ratio $\rho_{m} / \rho_{v \text { or } l}$. Here the subscripts $v$ and $l$ are the vapor and the liquid, respectively. By averaging the two kinds of the number of proton injection into cladding, $C_{v}$ and $C_{l}$, obtained by the two calculations, the final estimated number $C$ is deduced as follows.

$$
C=V R \times C_{v}+(1-V R) \times C_{l}
$$

In the annular flow model, the transport calculations of the protons were performed separately for the vapor or the liquid regions since the transformation of the geometry described in Section III. 2. (3) is cumbersome for the vapor region. In the vapor region, transport of a proton was evaluated only considering the ionization and the excitation and neglecting the Rutherford scattering. The transport is characterized by the linear tracks so that penetration point of a proton into the liquid film can be calculated uniquely with its $\boldsymbol{r}, \boldsymbol{u}_{p}, E$, and the stopping power. Actually, protons injected onto cladding penetrating the liquid films have enough energy so that the Rutherford scattering in the vapor region is insignificant for the energetic protons.

In the liquid film, the transport was calculated with SRIM-2008 as described in Section III. 2.

\section{Results and Discussion}

\section{Distribution of Recoil Protons}

Depth profiles of the recoil protons in the cladding injected by the process were calculated. The cumulative distributions of them by the homogeneous model are shown in Fig. 5.

Little difference is found between the distributions calculated for the different void ratio conditions. More than $75 \%$ of the protons stop at further than $1 \mu \mathrm{m}$ depth and $90 \%$ of them do within $30 \sim 40 \mu \mathrm{m}$ depth. The depth of them corresponds to the range $(R)$ of protons having energy of $0.18 \mathrm{MeV} 2.5 \mathrm{MeV}$. Since penetrating distance of a proton in the zirconium cladding is longer than the depth from the surface due to difference of the penetrating axis and the cladding normal one, the energy of the protons are larger than $180 \mathrm{keV}$ on the cladding surface.

The distribution of the initial energy of recoil protons fi-

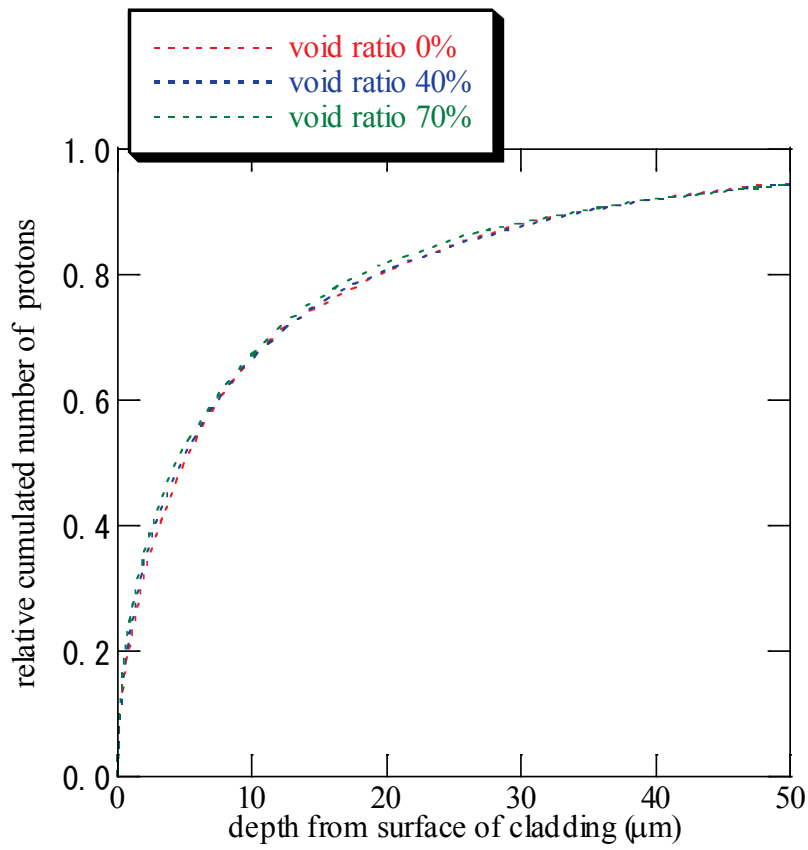

Fig. 5 Cumulated depth profiles of protons injected into cladding by $H(n, n)$ elastic scattering process calculated by the homogeneous model

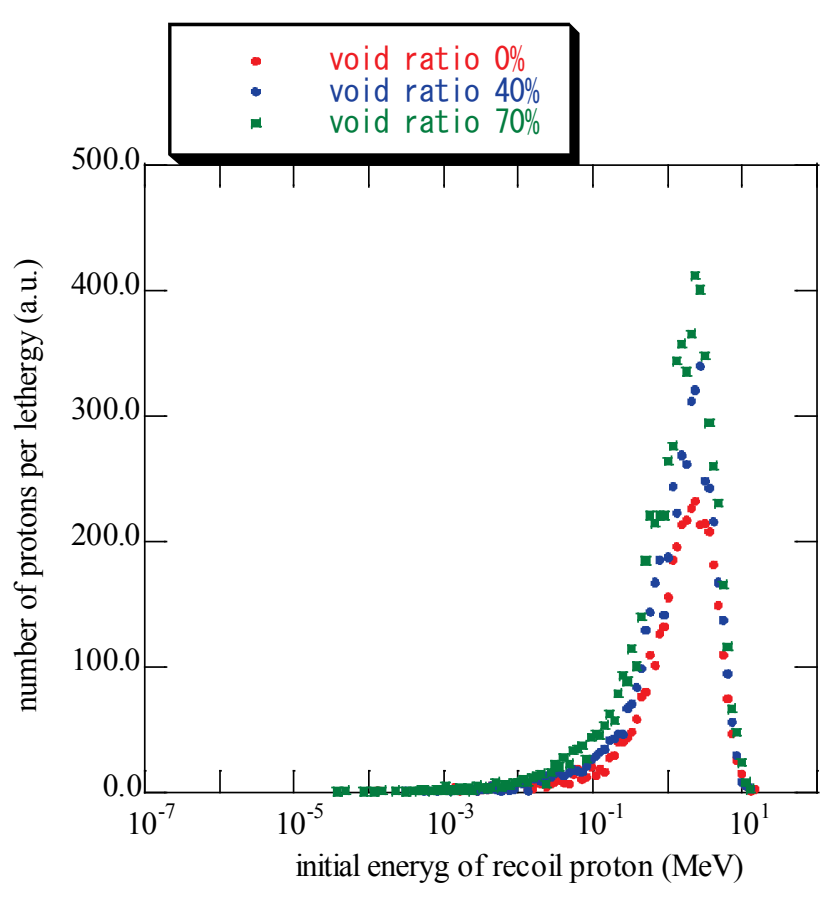

Fig. 6 Initial energy of protons at $H(n, n)$ reaction points in moderator calculated by the homogeneous model

nally injected into the cladding is shown in Fig. 6. The major part of them is found in an energy region from $0.1 \mathrm{MeV}$ to $10 \mathrm{MeV}$. The number of lower energy protons $(<1 \mathrm{keV})$ is quite low.

Considering the energy region described above $(0.18-$ $2.5 \mathrm{MeV}$ or $0.1-10 \mathrm{MeV})$, major process of the transport of 


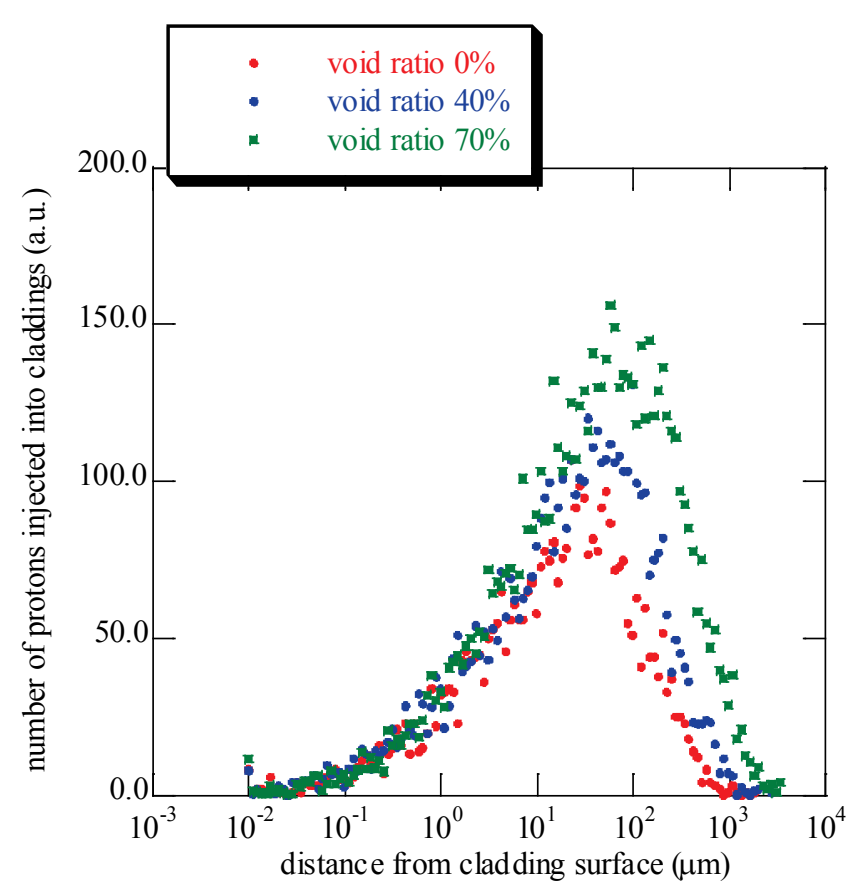

Fig. 7 Position of $H(n, n)$ reaction injecting proton into cladding calculated by the homogeneous model

the protons penetrating into the cladding is the ionization and the excitation, which characterized by the linear tracks. Therefore, transport of those protons can be simulated by the simpler model as done in the vapor region of the annular flow model in Section III. 3.

In Fig. 7, the distributions of distance from the $H(n, n)$ reaction position in the moderator to the cladding surface are shown for all the protons injected into the claddings. The major region spreads in $10 \mu \mathrm{m}$ to $1 \mathrm{~mm}$ from the cladding surface. The peak positions become further as the void ratio increases, because of increment of the proton range in the reciprocal relation to the density of the moderator.

\section{Increment of Hydrogen Content in Cladding}

We scored the number of protons injected into cladding in relation to the number of fission in the calculation geometry. Statistics of Monte Carlo calculations in the 51st generation are given for a typical case in Table 2. Almost $60 \%$ of neutron collisions with $\mathrm{H}$ generate recoil protons. The number of recoil proton emissions is almost 10 times larger than that of neutron source emissions. The probability of proton penetrating into the cladding is $0.69 \%$ per fission.

Assuming that the injected protons homogeneously dif-

Table 2 Number of particles and reactions used for calculations for $0 \%$ void ratio case

\begin{tabular}{lr}
\hline neutron source emission & 1003350 \\
neutron collision with H & 16953927 \\
recoil proton emission & 9846748 \\
proton jenjection into cladding & 3811 \\
fission in next generation & 551270 \\
\hline
\end{tabular}

Table 3 Increment of hydrogen content in cladding at burn-up of $40 \mathrm{MWd} / \mathrm{kgHM}$ by the $\mathrm{H}(\mathrm{n}, \mathrm{n})$ process

\begin{tabular}{llr}
\hline \multirow{2}{*}{$\begin{array}{llr}\text { void ratio } \\
(\%)\end{array}$} & $\begin{array}{l}\text { moderator } \\
\text { flow model }\end{array}$ & \multicolumn{1}{l}{$\begin{array}{l}\text { Increment of } \\
\text { H content }\end{array}$} \\
\hline 0 & homogeneous & 5.3 \\
\hline 40 & homegeneous & 7.6 \\
& annular & 7.9 \\
\hline 70 & homogeneous & 11.8 \\
& annular & 12.2 \\
\hline
\end{tabular}

fuse in the fuel cladding volume, the increment of $\mathrm{H}$ content in cladding per fission is deduced. We calculated the number of fission corresponding to the burn-up unit and deduced the increment of $\mathrm{H}$ content for the target burn-up by the process neglecting the change of properties of fuel rods (the isotopic composition of pellets, the oxide layer, and the cruds). The results at burn-up of $40 \mathrm{MWd} / \mathrm{kgHM}$ are listed in Table 3 .

The effect of the moderator modeling (homogeneous and annular flow) on the increment is less than $5 \%$ so that the homogeneous flow model is considered adequate as well as the annular flow one to evaluate the content. The increment varies with the void ratio from 5.3 to $11.8 \mathrm{wt}-\mathrm{ppm}$. In the higher void ratio cases, the range of proton in the moderator increases so that lower energy protons generated by the $\mathrm{H}(\mathrm{n}, \mathrm{n})$ reactions at further positions can reach the cladding as shown in Fig. 7. Accordingly the $\mathrm{H}$ content by the process increases in such cases.

\section{Burn-up Effect}

The isotopic composition of nuclear fuels changes with burn-up, which affects the neutron transport in the fuel cell. We evaluated the composition by burn-up calculations with MVP-II. ${ }^{7)}$ Library u4cm6fp50bp16T was used for the decay chain data and the linear power rate of the rod was set $18.2 \mathrm{~kW} / \mathrm{m}$ so that burn-up of $55 \mathrm{MWd} / \mathrm{tHM}$ is achieved after 65 months continuous operation. Taking the composition into account, sequential calculations of MCNP-5 and SRIM-2008 were performed for void ratio $=0 \%$ condition.

Results are summarized in Table 4. As the burn-up increases, the number of recoil protons injected into the cladding per power increased up to 1.65 times larger than that of the fresh fuel case. This is due to the increment of the total neutron flux to compensate decrement of the macroscopic fission cross section of the fuel rod monotonously with burn-up.

Table 4 Proton injection rate into cladding in relation to fuel burn-up

\begin{tabular}{rr}
\hline $\begin{array}{l}\text { burn-up } \\
(\mathrm{MWd} / \mathrm{kgHM})\end{array}$ & $\begin{array}{l}\text { injection of protons into } \\
\text { cladding per fission } \\
\text { heat (proton / J) }\end{array}$ \\
\hline 0 & $2.14 \mathrm{E}+14$ \\
11 & $2.54 \mathrm{E}+14$ \\
33 & $3.09 \mathrm{E}+14$ \\
55 & $3.53 \mathrm{E}+14$ \\
\hline
\end{tabular}


In the simulation, influence of neighboring fuels is neglected. Practically, highly irradiated fuels are loaded with low irradiated ones in a core. Thus the neutron flux might not increase in the former even in higher burn-up cases. Therefore, the number of proton injections might be influenced by the fuel rods surrounding the focused one. A more detailed simulation is required considering power and void history data of the focused and the surrounding fuel rods.

Besides, we neglected the cruds and the oxide layer deposited on the surface with burn-up. Recoil protons certainly penetrate into them, but it is unclear how the injected hydrogen atoms diffuse to the whole volume of the cladding. Thus, further study is required in conjunction with material property of the cruds and the oxide layer.

\section{Summary and Conclusion}

In order to clarify a part of hydrogen absorption mechanisms in fuel claddings, we firstly focused on a process that recoil protons by neutron elastic scatterings with hydrogen atoms in the moderator of LWR penetrate into the claddings. To simulate transports of the neutron and the proton one by one, we established the calculation scheme combining MCNP-5 and SRIM-2008.

We estimated the number of the protons injected into claddings for a simplified BWR calculation geometry in which the cruds and the oxide layer on the outer surface of the cladding are neglected. Calculation results show the number of recoil protons is 10 times larger than that of fission neutron emissions and the $\mathrm{H}$ injection rate into cladding is $0.69 \%$ of fission rate in the $0 \%$ void ratio case. The rate was found increasing in the cases of higher void ratio and higher burn-up assuming the constant pin power rate. Neglecting that the fuel property changes with burn-up, the increment of $\mathrm{H}$ content in claddings by the process is estimated 5 12 wt-ppm at $40 \mathrm{MWd} / \mathrm{kgHM}$ burn-up, which cor- corresponds to $5 \sim 20 \%$ of hydrogen content measured for conventional irradiated fuel claddings. Therefore, it is essential to consider the process when we compare in-pile and out of pile tests for $\mathrm{H}$ content.

Since $\mathrm{H}$ atoms in $\mathrm{Zr}$ are considered to diffuse in whole volume of the cladding, 3-D calculations in which void ratio axially varies should be done to quantify the increment of the $\mathrm{H}$ content in it. Information of the power history of the fuel rod is also important. The role of the soft and the hard cruds and the oxide layer for the focused process should be investigated further.

\section{References}

1) H. J. Sell, S. Trapp-Pritsching, F. Garazarolli, "Effect of alloying elements and impurities on in-BWR corrosion of zirconium alloys," Proc. Fourteenth Int. Symp. Zirconium in the nuclear industry, Stokholm, Sweden, June 13-17, 2004, 404 (2004).

2) K. Suyama, J. Katakura, Y. Ohkawachi et al., Libararies based on JENDL-3.2 for ORIGEN2 code : ORLIBJ32, JAERI-Data/Code 99-003, Japan Atomic Energy Research Institute (JAERI) (1999), [in Japanese].

3) X-5 Monte Carlo Team, MCNP $P^{T M}$ a general Monte Carlo n-particle transport code, version 5, LA-UR-03-1987, Los Alamos National Laboratory (LANL) (2003).

4) J. F. Ziegler, J. P. Biersack, M. D. Ziegler, SRIM the stopping and range of ions in matter, version 5, LuLu.com (2008).

5) R. E. MacFarlane, D. W. Muir, The NJOY Nuclear Data Processing System, Version 91, LA-12740-M, Los Alamos National Laboratory (1994).

6) K. Shibata, T. Kawano, T. Nakagawa et al., "Japanese evaluated nuclear data library version 3 revision-3: JENDL-3.3," $J$. Nucl. Sci. Technol., 39[11], 1125-1136 (2002).

7) Y. Nagaya, K. Okumura, T. Mori et al., MVP/GMVP II: general purpose Monte Carlo codes for neutron and photon transport calculations based on continuous energy and multigroup methods, JAERI 1348, Japan Atomic Energy Research Institute (JAERI) (2005). 